\title{
Adaptive, integrated sensor processing to compensate for drift and uncertainty: a stochastic 'neural' approach
}

\author{
T.B. Tang, H. Chen and A.F. Murray
}

\begin{abstract}
An adaptive stochastic classifier based on a simple, novel neural architecture - the Continuous Restricted Boltzmann Machine (CRBM) is demonstrated. Together with sensors and signal conditioning circuits, the classifier is capable of measuring and classifying (with high accuracy) the $\mathrm{H}^{+}$ion concentration, in the presence of both random noise and sensor drift. Training on-line, the stochastic classifier is able to overcome significant drift of real incomplete sensor data dynamically. As analogue hardware, this signal-level sensor fusion scheme is therefore suitable for real-time analysis in a miniaturised multisensor microsystem such as a Lab-in-a-Pill (LIAP).
\end{abstract}

\section{Introduction}

Rapid progress in both Lab-on-a-Chip (LOC) and Systemon-Chip (SoC) technologies has encouraged increasing interest in electronic health care [1]. Applications have ranged from telemedicine to bioanalysis, from patient monitoring to implantable devices. In conjunction with image scanning [2], several other biomedical instruments provide constant monitoring of such essential physiological parameters as temperature, $\mathrm{pH}$, oxygen and pressure [3-6].

It is clearly desirable that such instruments be integrated and thus miniaturised, although it is inherently more difficult to extract useful information from what are now far more noisy and unstable measurements. With integration, however, comes the possibility of sensor redundancy (multiple sensors of the same or different types). There is, therefore, a need for robust, adaptive algorithms for sensor fusion and early pre-processing, that can be implemented directly in hardware, with low power consumption. In particular, algorithms that can process continuous-time, analogue sensor signals directly are especially useful [7].

This paper investigates the ability of stochastic neural computation to fuse multisensor data at signal level under conditions of significant sensor drift. Figure 1 shows two separate sets of measured, and typical, sensor drift (over $21 \mathrm{~h}$ ) in $10 \mathrm{pH}-$ ISFET sensors. The data is obtained by measuring the potential of several drifting reference electrodes in a neutral ( $\mathrm{pH}$ 7) buffer over time. The drift, which causes the drop in reference potential, is due to the dissolution of $\mathrm{AgCl}$ from the reference electrode [8]. In real applications, this drift will destroy the capabilities of the sensor system unless regular recalibration, or some form of adaptive self-calibration, is introduced. We are interested in the latter approach. An adaptive classifier must be able both to track sensor drift and to maintain its classification ability

\section{(C) IEE, 2004}

IEE Proceedings online no. 20040213

doi:10.1049/ip-nbt:20040213

Paper first received 14th November 2003 and in revised form 13th January 2004 The authors are with the School of Engineering and Electronics, The University of Edinburgh, Edinburgh, United Kingdom in the absence of a complete, representative training set (i.e. only data drawn from a sub-class of the full data space are likely to be available at one time, under normal operating conditions). This is a serious challenge. Without constrained
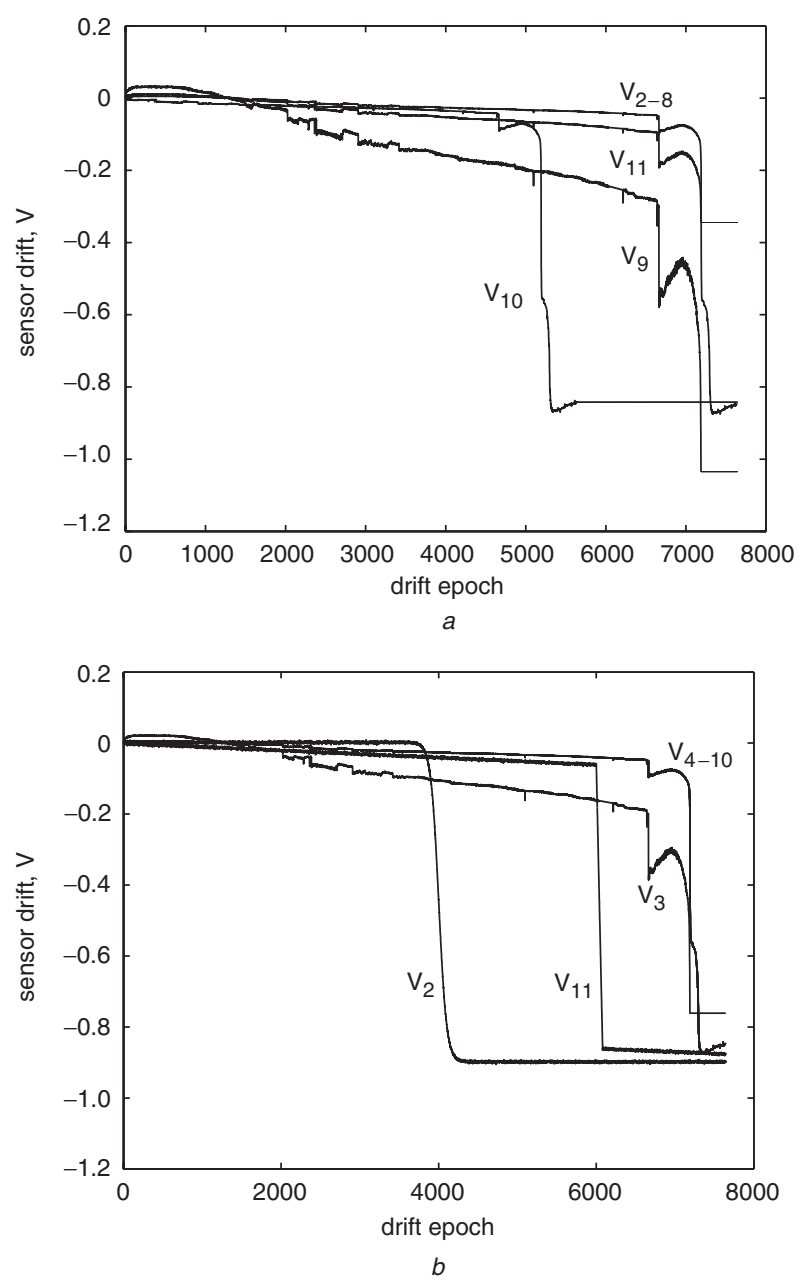

Fig. 1 Two separate sets of sensor drift in several reference electrodes over time 
training, most continuously-adaptive systems will simply "learn" to model the current distribution, losing the ability to model the entire data space completely. Such systems thus lose classification ability abruptly and completely.

In this paper, we describe the adaptive stochastic classifier, and discuss training and some important practicalities in this application. Experimental results that demonstrate the feasibility of this approach and compare its performance with both linear and nonlinear (multi-layer perceptron) classifiers are presented. Both are also "neural" architectures that can be trained to classify data, but are not continuously-adaptive [9].

\section{Architecture}

The classifier's operation can be viewed in two stages; unsupervised feature extraction and supervised (linear) classification. Our aim is to render the feature-extraction stage adaptive and able to present a consistent set of features to the supervised classifier in the presence of drift and noise. The Continuous Restricted Boltzmann Machine (CRBM) [10] is a generative model that is capable of a form of autonomous feature extraction and is based upon Hinton's product-of-experts architecture [11]. The CRBM has one visible layer that passes and receives data to and from the outside world and one hidden layer that underpins the ability to model the mechanisms that underlie a set of data (Fig. 2). This algorithm is specifically designed to accept analogue continuous signals and to build from them a continuous-valued generative model.

In the absence of a full physical/chemical/biological model, a generative model is a useful statistical (often "neural") model that is trained to generate, or to "model" data with the same statistical structure as a set of "training data". A good generative model mimics both the structure and inherent noisiness of real, multi-dimensional data. It is usable for classification and novelty detection of unseen data drawn from the same physical source as the training data, as the model can be viewed as providing an "explanation" of the mechanism(s) that generated the data.

The CRBM thus avoids quantisation and the loss of information in, for example, the binary Restricted Boltz- mann Machine (RBM) [11, 12]. Furthermore, the CRBM's simple computation requires only local addition and multiplication, and is thus (analogue) hardware amenable [10]. The CRBM is trained in an unsupervised manner, adapting both its weights (internal model parameters) and internal noise sources by minimising contrastive divergence $[10,11]$.

The final, output, block of the classifier used in this paper is a single layer perceptron (SLP). In our application, there is one temperature sensor [13] and $10 \mathrm{pH}$-ISFET sensors [14] which measure the environmental concentration of $\mathrm{H}^{+}$ ions. The temperature sensor monitors the ambient temperature, to improve overall system robustness, as the $\mathrm{pH}$ sensors are also temperature-dependent. The use of redundant $\mathrm{pH}$-ISFET sensors aims to improve the overall robustness of the classifier. Each sensor output is passed directly to a visible unit $i$ in the CRBM. The 11 visible units are connected to and from the neurons $j$ in the hidden layer via a symmetrical weight matrix $\mathbf{w}_{i j}$. In addition, two permanently-on bias, or "threshold" units $V_{0}$ and $H_{0}$ encode, in the weights that lead from them, the adaptive thresholds, or biases, of the hidden and visible units respectively. It will be seen that much of the modelling ability is found in these weights. In the hidden layer, a total of 4 hidden units/neurons aim to encode the mechanisms behind the sensor data distribution. Smaller numbers of hidden units result in poorer models, with too few degrees of freedom. Although more hidden units offer the promise of a better model, we wish to minimise computation and therefore network size. Furthermore, Occam's Razor suggests that a parsimonious model is most likely to be useful and informative [15]. The activity for each stochastic neuron is given by:

$$
s_{j}=\tanh \left(a_{j} \cdot\left(\sum_{i} \mathbf{w}_{i j} s_{i}+\sigma \cdot N_{j}(0,1)\right)\right)
$$

where

$$
\begin{aligned}
s_{i}= & \text { input from neuron } i \\
a_{j}= & \text { noise control parameter: specific to each (visible } \\
& \text { or hidden) unit }
\end{aligned}
$$

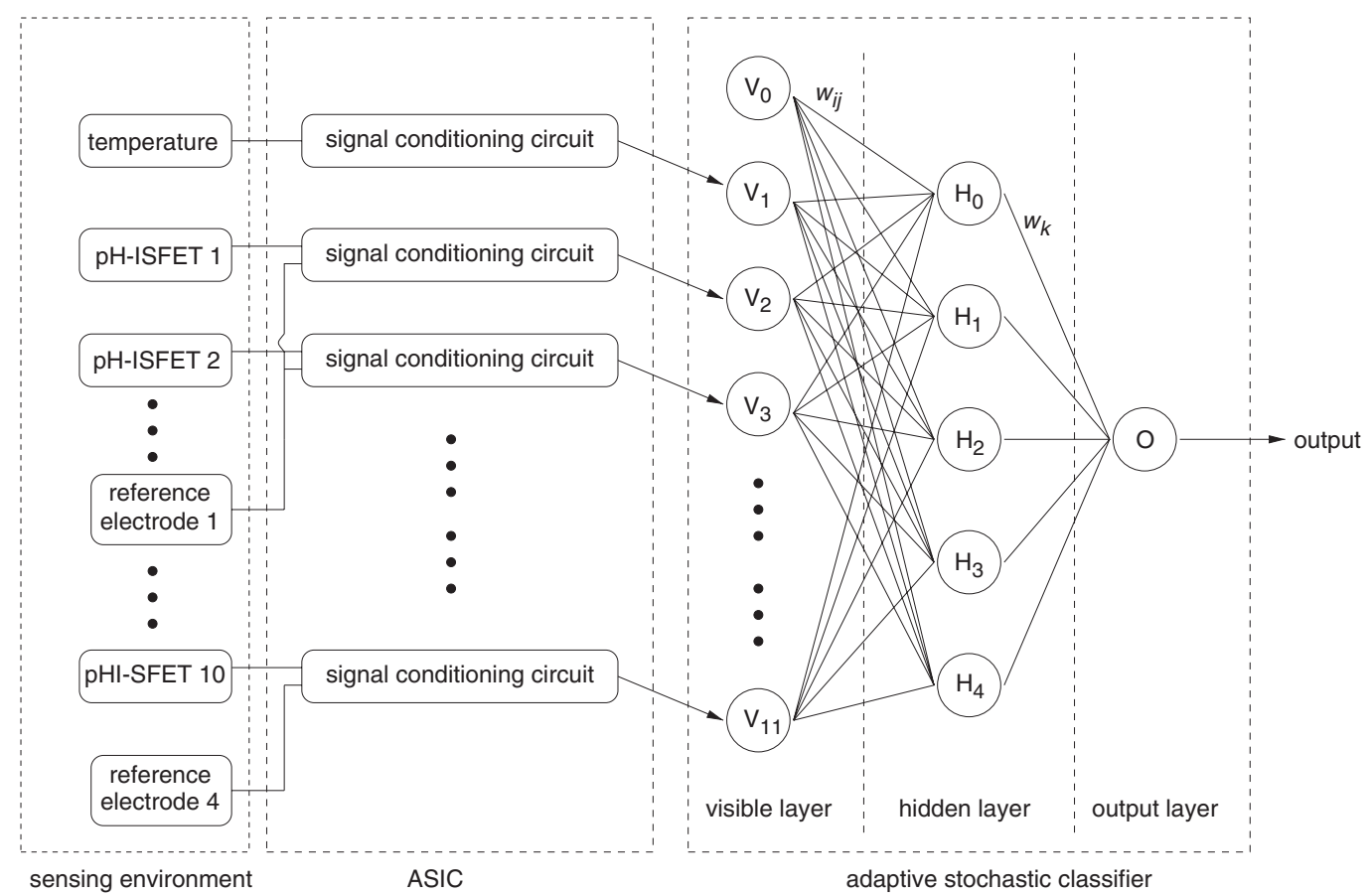

Fig. 2 An adaptive stochastic classifier in a typical multisensor microsystem with 11 visible, 4 hidden, 2 bias and 1 output units 
$\sigma=$ noise scaling constant: specific to each (visible or hidden) layer

$N_{j}(0,1)=$ sampled from unit-magnitude, zero-mean Gaussian noise source

There are three sets of learning parameters in the classifier. They are the weights $\mathbf{w}_{i j}$, the noise control parameters, $a_{j}$ of the CRBM and the weights $\mathbf{w}_{k}$ in the output SLP layer. The CRBM parameters are optimised by minimising contrastive divergence (MCD) [11] while the SLP is trained using the delta rule [16].

\section{Methodology}

The signal conditioning circuits can be tuned to maximise sensor sensitivity and thus the separation of any clusters in the sensor data. This facilitates data modelling. However, variance in the threshold voltage of the $\mathrm{pH}$-ISFET sensors due to the fabrication process means that very few integrated sensors of this type can respond linearly across the full $\mathrm{pH}$ range. A compromise is therefore made between sensor sensitivity and linearity that renders these integrated sensors slightly nonlinear. The CRBM is fundamentally a nonlinear model and is able to deal with at least this level on nonlinearity, as will be demonstrated. Based on measurements [17], a particular $\mathrm{pH}-\mathrm{ISFET}$ sensor, when immersed in a solution with $\mathrm{pH} x$, can be modelled as an output voltage $y=A-B x(\mathrm{mv})$ with a correlation coefficient ${ }^{1}$ $R^{2}=0.98$. Typically, $A=1733.66$ and $B=43.51$. Within an array of pH-ISFET sensors, threshold voltages vary, the constant term $A$ varies, while the sensitivity $B$ remains fairly consistent. The temperature sensor can be modelled with an output voltage of $y=34.86 T-125.10(\mathrm{mv})$ for temperature $T$ with $R^{2}=0.99$.

Figure 3 shows the training process for the adaptive stochastic classifier. Initially, the CRBM is trained with 2 datasets. Dataset A comprises measurements on a solution with a temperature of $37^{\circ} \mathrm{C}$ and $\mathrm{pH}$ 4. Dataset $\mathrm{B}$ consists of

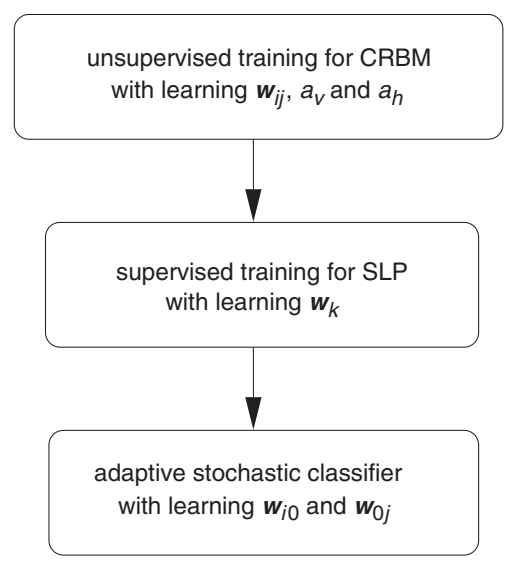

Fig. 3 Sequential training for the adaptive stochastic classifier

measurements on a solution with a temperature of $37^{\circ} \mathrm{C}$ and $\mathrm{pH}$ 10. To facilitate training, the noise scaling constant $\sigma$ is set to a value that avoids both the over-fitting that is associated with low noise and the complete loss of modelling ability that is produced by high noise. Empirical experiments lead to optimal values of $\sigma$ of 0.2 for visible

${ }^{1}$ In this context, correlation coefficient is a quantity which gives the quality of a least squares fitting to the measurements. units and 0.4 for hidden units. These optimal values are problem-dependent.

The learning rate for the CRBM must also be considered carefully. Our empirical "rule of thumb", drawn from several CRBM-modelling projects, is to have the visible noise control parameters' learning rate $\eta_{v} 10$ times that of hidden units and weights, $\eta_{h}$ and $\eta_{w}$, respectively. This encourages autonomous annealing through adaptation of the visible layer's noise control parameters $a_{v}$ on a shorter timscale than that for adaptation of the weights $\mathbf{w}_{i j}$ and hidden noise control parameters $a_{h}$ to model the detail of training data distribution.

"Greedy training" is used to train the CRBM and SLP layers. CRBM training is allowed to reached equilibrium and is then stopped. At this stage, the SLP is untrained. The SLP is then trained to map the activity of the CRBM hidden layer $\left(H_{0-4}\right)$, as the input data is presented to the visible layer, to the known-correct SLP output classification. This training is performed with (a) the visible units clamped to datasets A and B in the training set, (b) the output units clamped to the corresponding labels and (c) fixed weight $\mathbf{w}_{i j}$, noise control parameters $a_{v}$ and $a_{h}$. The only learning parameters are the weights $\mathbf{w}_{k}$ for the output unit.

After training, the learning rates for all parameters except the CRBM bias weights are set to zero. This setting allows the classifier to adapt to sensor drift via $\mathbf{w}_{i 0}{ }^{2}$ and suppresses the competitive learning in CRBM that would otherwise generate a totally new distribution (in this case, one cluster instead of two) and hence destroy classification. Secondly, it causes the representations (the activities of hidden units) that are the extracted "features" passed to the subsequent layer to be consistent for a particular dataset in the presence of drift. Equation (1) shows that the hidden unit's state $s_{j}$ drifts as the sensor output $s_{i}$ drifts. Therefore, the visible bias unit's weight $\mathbf{w}_{0 j}$, which encodes the biases for all the hidden units, must be allowed to adapt to compensate. $\Delta w_{i 0}$ accounts for a shift in the mean of data distribution, so

$$
w_{0 j}(t)=w_{0 j}^{*}-\eta_{w} \sum_{i}\left(w_{i j}^{*}\left\{w_{i 0}^{*}-w_{i 0}(t)\right\}\right)
$$

where $i \neq 0$ and $j \neq 0 . w^{*}$ refers to weight after the CRBM is trained and before it is exposed to drifting data. Hence the weight change for a visible bias unit at time $t+1$ is:

$$
\Delta w_{0 j}(t+1)=\eta_{w} \sum_{i}\left(w_{i j}^{*}\left\{w_{i 0}(t+1)-w_{i 0}(t)\right\}\right)
$$

where $i \neq 0$ and $j \neq 0$.

\section{Experimental results and discussion}

The classifier has been trained with two datasets (each with 400 samples) using real sensor data. The CRBM and the SLP are trained for 3000 and 500 epochs respectively. Training results are discussed in section 4.1, while section 4.2 discusses the classifier's ability to track sensor drift via constrained on-line adaptation of the CRBM. The experiment in section 4.2 is conducted by introducing a typical pattern of drift to the dataset $\mathrm{A}$ and presenting the data to the visible units of the CRBM. The data is taken over a period of $76440 \mathrm{~s}$, sampled at $0.1 \mathrm{~Hz}$. There are therefore 7644 "drift epochs" in the experiment. Note that no samples from dataset $\mathrm{B}$ are presented to the classifier for the experiment in section 4.2 .

${ }^{2}$ Experiment [10] has shown that the hidden bias unit's weights acts as an encoder for the mean of the training data distribution. This is due to its state (permanently ' +1 ') which allows it to learn faster (with a larger weight change $\left.\Delta w_{i 0}\right)$ than other hidden units that have near-zero initial states. 


\subsection{Learning to classify}

Figure $4 a$ and $b$ show 2-dimensional plots of sensor signals for $V_{1}$ (temperature sensor), $V_{2}$ (pH-ISFET sensor 1) and $V_{3}$ (pH-ISFET sensor 2). Sensor noise causes significant overlap between the two clusters in all 11 dimensions. Figure $4 c$ shows the evolution of the CRBM's parameters $a_{v}$ which suggest that training equilibrium is reached within 3000 training epochs.

It is a characteristic of the CRBM that the stochastic hidden units can adapt to become more or less binary [10]. After training, hidden unit $\mathrm{H}_{2}$, in this particular case, has a large noise control parameter $\left(a_{h}=2.1\right)$ and thus behaves as a binary "decision" unit that captures gross structure in the data while other hidden units' $a_{h}$ remain approximately 1 , rendering their behaviour more deterministic. More deterministic units are able to model finer detail in the data distribution. Similarly, the output unit's weight $\mathbf{w}_{k}$ connecting to the hidden unit $\mathrm{H}_{2}$ has increased significantly to 6.2 after 500 training epochs, as shown in Fig. $4 e$.

These parameters hold the clue as to how the CRBM models these two 11-dimensional clusters of data. The large values imply that the activities of hidden unit $\mathrm{H}_{2}$ and output unit are very sensitive to the particular elements in the sensor data space. The trained CRBM is then tested with two new datasets (400 samples for each sub-class). As indicated in Fig. $4 f$, the clear separation between the output response for the two datasets yields $100 \%$ accurate classification by simply thresholding at zero. To investigate the classifier's performance further, the above experiment has been repeated using solutions with different $\mathrm{pH}$ values in pairs. Each pair has one acidic and one alkaline solution. Figure $5 a$ shows the corresponding output unit's response. In the worst case (i.e. small separation between data clusters), the first solution (dataset A) has a pH of 6 while the second (dataset B) has $\mathrm{pH}$ 8. A 2-dimensional plot of sensor outputs for visible units $V_{1}$ and $V_{2}$ is shown in Fig. $5 b$. Despite overlap between the data clusters, the CRBM/SLP still classifies with $81.75 \%$ accuracy, as shown in Fig. $5 c$ and $d$, as the CRBM's ability to model the shape and spread of a data cluster captures structure and "shapes" in the data that a simple linear or nonlinear classifier alone does not.

\subsection{Tracking sensor drift}

As shown in Fig. $1 a$, there are three interesting phases in this temporal data stream. The first phase ends at the $4000^{\text {th }}$ drift epoch where the classifier must adapt to gradual sensor drift for each sensor output. The second phase ends at the $6000^{\text {th }}$ drift epoch when the ninth $\mathrm{pH}$-ISFET sensor $\left(V_{10}\right)$ fails catastrophically. Finally, all remaining pH-ISFET sensors fail around the $7644^{\text {th }}$ drift epoch.

Figure $6 a$ shows the evolution of $\mathbf{w}_{i 0}$ which "follows" the sensor drift. The CRBM section of the classifier is compensating autonomously for the drift. However, around the $7644^{\text {th }}$ drift epoch, there is a major shift in several sensor outputs and a failure in classification, indicating that the CRBM model has broken down completely and is now, quite correctly, responding to the input signal as completely new data. This is obviously caused by simultaneous failure in all remaining $\mathrm{pH}$-ISFET sensors.

Figure $6 b$ shows the activity of the output unit as sensor drift occurs and the CRBM's constrained adaptation responds to the gradual change in sensor activity. Ideally, a straight line (output =1) should be obtained, as only dataset A is presented. However, any sudden and significant increase in the speed of drift will result in a temporary loss of accurate classification as the CRBM adapts to take
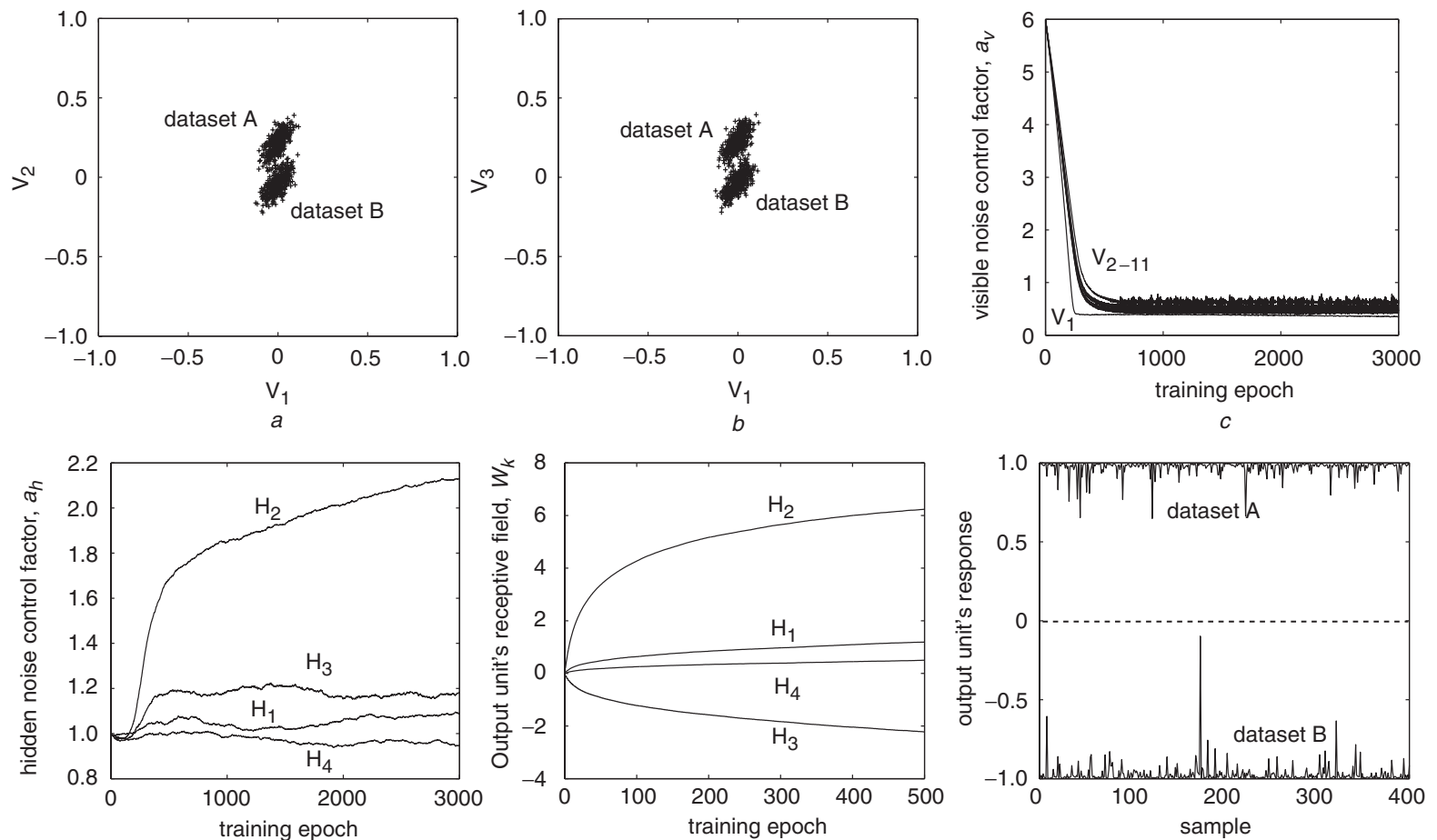

$c$

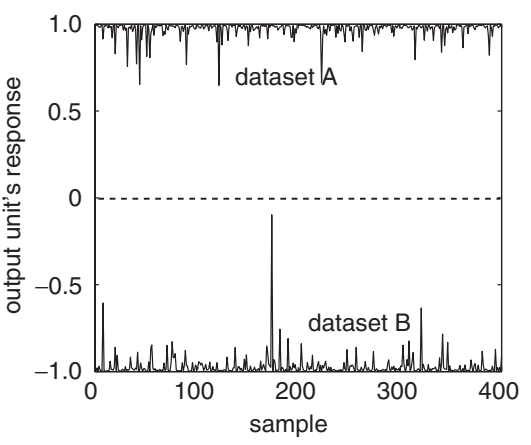

d

e

$f$

Fig. 4 Learning in the classifier

$a$ The training data for visible units $V_{1}$ and $V_{2}$

$b$ The training data for $V_{1}$ and $V_{3}$

$c$ The visible noise control parameter $a_{v}$ for CRBM over 3000 training epochs

$d$ The hidden noise control parameter $a_{h}$ for CRBM over 3000 training epochs

$e$ The weights $\mathbf{w}_{k}$ for the output unit in SLP over 500 training epochs

$f$ The output unit's response with respect to datasets $\mathrm{A}$ and $\mathrm{B}$. The learning rates are 0.1 for weight vector and 3 for noise control parameters 

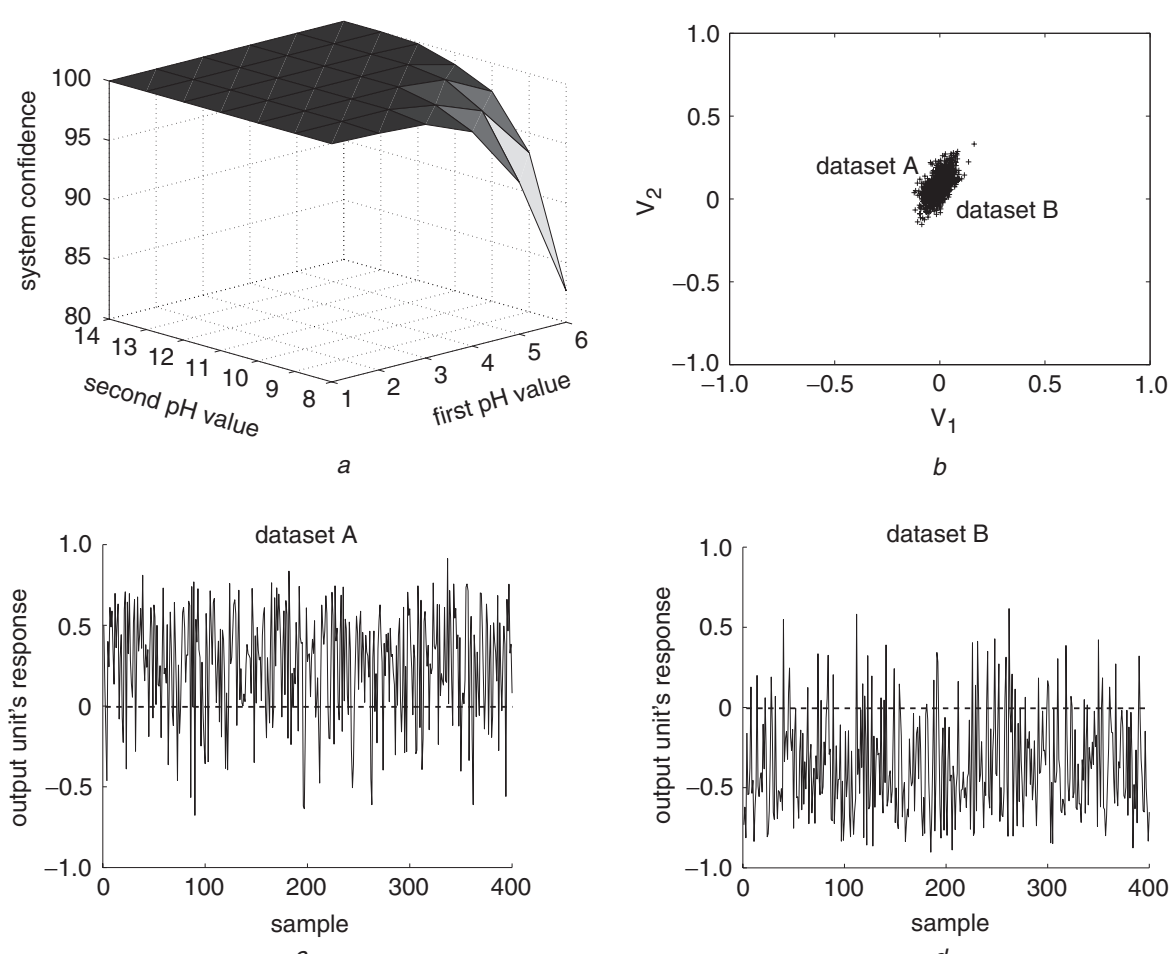

Fig. 5 Classifier performance over resolution

$a$ The compiled result for various pairs of solutions

$b$ The training data for visible units $V_{1}$ and $V_{2}$ with first $\mathrm{pH}=6$ (dataset A) and second $\mathrm{pH}=8$ (dataset $\mathrm{B}$ )

$c$ The output unit's response for dataset $\mathrm{A}$ in the worst case

$d$ The output unit's response for dataset $\mathrm{B}$ in the worst case
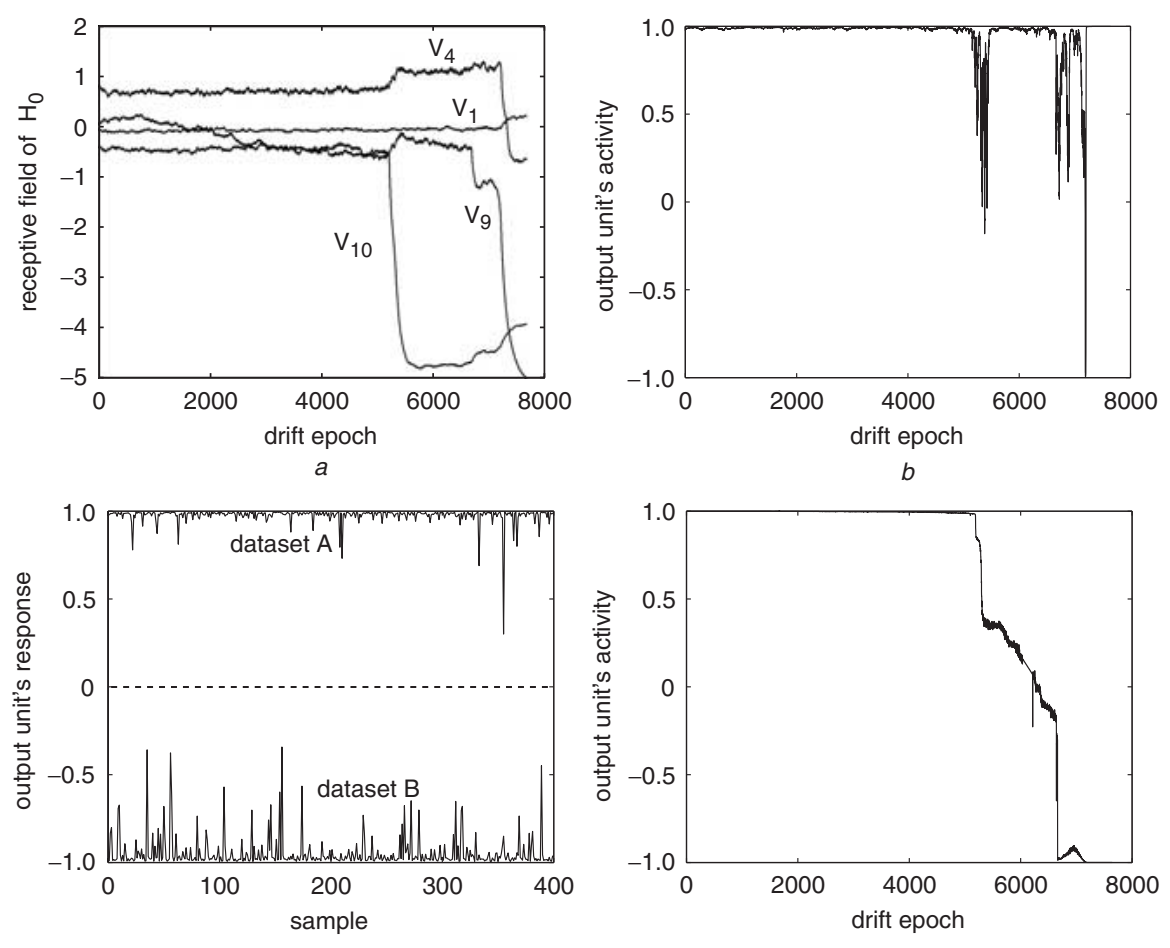

Fig. 6 Evolution of $\boldsymbol{w}_{i 0}$, activity of output unit as sensor drift occurs, classifier response at $6000^{\text {th }}$ drift epoch and MLP classifier response during 7644 drift epochs

$a$ Weight change for hidden bias unit $\mathbf{w}_{i 0}$

$b$ The output unit's activity during the 7644 drift epochs

$c$ The classifier's response to the two datasets at $6000^{\text {th }}$ drift epoch

$d$ MLP classifier's output response during the 7644 drift epochs.

Experiment is carried out based on drift data shown in Fig. $1 a$

account of the speedier change. This is due to the use of a constant learning rate for the CRBM- a common problem for on-line learning in a dynamic environment [18-20]. Otherwise, the classifier is working as intended and well.
Figure $6 c$ shows its response to two datasets (400 new samples for each sub-class) at the $6000^{\text {th }}$ drift epoch. $100 \%$ classification accuracy is achieved very simply by thresholding the SLP output at zero. 
To highlight the significance of the classifier's on-line unsupervised learning, trained, but subsequently nonadaptive, linear (another SLP) and MLP classifiers are used as benchmarks. The SLP is trained with the two original datasets A and B (i.e. without drift data) for 500 training epochs. It has a learning rate of 0.10 and a sigmoidal activation function. The trained SLP cannot classify even the initial data with $100 \%$ accuracy, highlighting the (modest) inherent nonlinearity of the classification task. The classification ability subsequently collapses as sensor drift occurs. The comparison in performance is summarised in Table 1. The MLP has 15 hidden and 1 output units. All units have sigmoidal activation function and use a back-propagation (BP) gradient descent with momentum learning rule [21]. The learning rate is 0.05 and the momentum factor is 0.9. The MLP is trained with the two original datasets for 20000 learning epochs and achieves a mean square error (MSE) of $4.92 \times 10^{-4}$, before being

Table 1 Classifiers' output and accuracy at various drift epochs for drift data in Fig. 1a

\begin{tabular}{llccc}
\hline \multirow{4}{*}{ Parameter } & \multicolumn{4}{c}{ Drift epochs } \\
& Method & $4000^{\text {th }}$ & $6000^{\text {th }}$ & $7644^{\text {th }}$ \\
\hline Output & Linear & 0.78 & -0.18 & -1.00 \\
& MLP & 0.99 & 0.15 & -1.00 \\
& CRBM+SLP & 0.98 & 0.96 & 1.00 \\
Accuracy & Linear & 99.875 & 68.000 & 50.000 \\
(\%) & & & & \\
& MLP & 99.625 & 77.375 & 50.000 \\
& CRBM+SLP & 99.875 & 95.500 & 50.000 \\
\hline
\end{tabular}

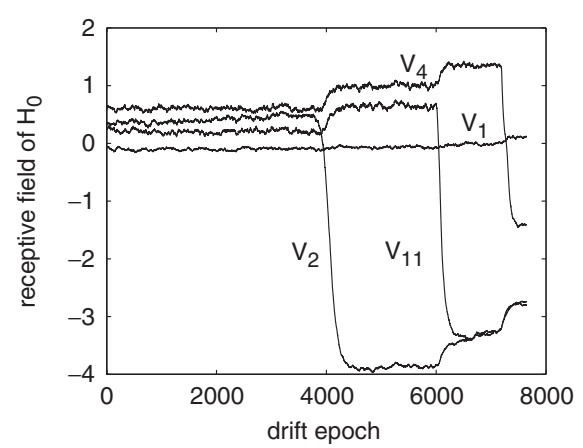

a

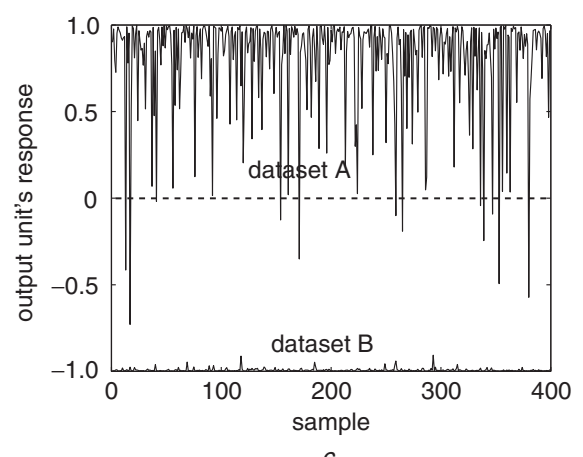

c exposed to drifting data. The output unit's activity is recorded throughout the drift epochs by Fig. $6 d$. The MLP's classification ability also collapses during sensor drift, while the CRBM maintains $95.5 \%$ correct classification during gentle sensor drift (up to the $6000^{\text {th }}$ epoch). Naturally, all classifiers fail when the sensor activity changes dramatically around $7600^{\text {th }}$ epochs.

The above experiment is repeated with a second set of drift data (Fig. 1b) to confirm that the above result is not an accident. The datastream is divided into four phases. The first ends at the $3500^{\text {th }}$ drift epoch where the classifier is adapting to gradual sensor drift. The second phase ends at the $5000^{\text {th }}$ drift epoch when the first pH-ISFET sensor $\left(V_{2}\right)$ fails. The third phase ends at the $6400^{\text {th }}$ drift epoch when the tenth $\mathrm{pH}$-ISFET sensor $\left(V_{11}\right)$ fails. The remaining drift epochs then form the last phase. The experimental results are shown in Fig. 7. As in the previous experiment, the CRBM/SLP classifier compensates for sensor drift as well as sensor failure if sufficient time for adaptive recovery is allowed. If several sensors fail simultaneously or within a very short time (with respect to the learning rate), the CRBM/SLP classifier will fail. Table 2 summarises the results of this second experiment, highlighting once more the CRBM's ability to "track" sensor drift that destroys the performance of other trained, but nonadaptive, classifiers.

\section{Conclusions}

The implementation of an adaptive classification system has been presented in the context of an 11-dimensional microsystem application. The results show that a CRBM with 4 hidden units is able to track nonlinear sensor drift and to classify noisy sensor data accurately and for far

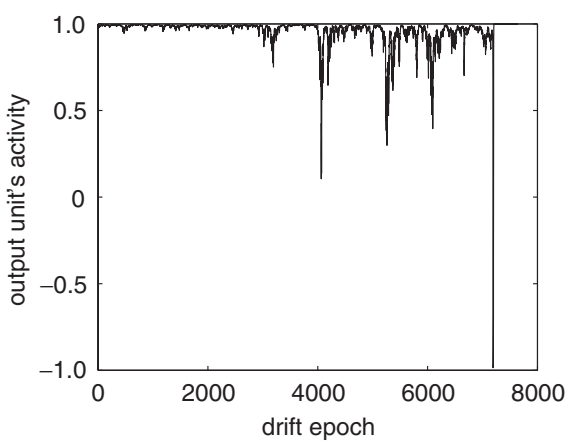

$b$

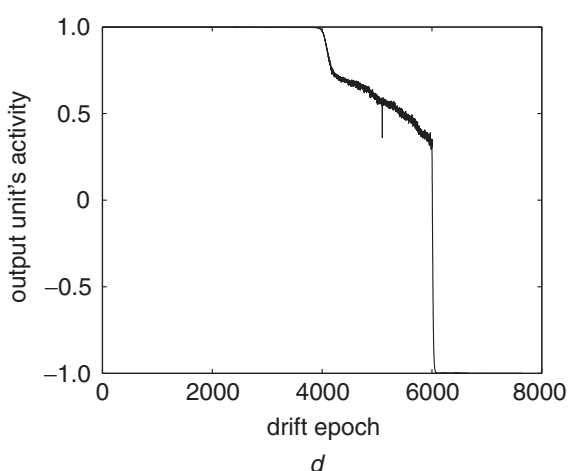

Fig. 7 Evolution of $\boldsymbol{w}_{i 0}$, activity of output unit as sensor drift occurs, classifier response at $6400^{\text {th }}$ drift epoch and MLP classifier response during 7644 drift epochs

$a$ Weight change for hidden bias unit $\mathbf{w}_{i 0}$ during the 7644 drift epochs

$b$ The output unit's activity during the 7644 drift epochs

$c$ The classifier's response to the two datasets at the $6000^{\text {th }}$ drift epoch

$d$ MLP classifier's output response during the 7644 drift epochs.

Experiment is carried out based on drift data shown in Fig. $1 b$ 
Table 2 Classifiers' output and accuracy at various drift epochs for second set of drift data in Fig. $1 b$

\begin{tabular}{lllll}
\hline \multirow{2}{*}{ Parameter } & Method & $3500^{\text {th }}$ & $5000^{\text {th }}$ & $6400^{\text {th }}$ \\
\hline \multirow{2}{*}{ Output } & Linear & 0.87 & 0.02 & -0.85 \\
& MLP & 1.00 & 0.59 & -1.00 \\
& CRBM+SLP & 0.99 & 0.90 & 0.92 \\
Accuracy & Linear & 99.875 & 76.000 & 50.750 \\
(\%) & & & & \\
& MLP & 100.000 & 84.250 & 50.000 \\
& CRBM+SLP & 99.875 & 96.500 & 98.500 \\
\hline
\end{tabular}

longer than does a carefully-trained, but non-adaptive neural classifier. Most importantly, the CRBM can be configured to respond sensibly to incomplete and "unbalanced" real-time input data that do not adequately represent the distribution of the training data.

We have also studied the classifier's ability to cope with major faults in the sensors. It has been tested rigorously with noisy real data including sensor outputs that drift at diverse rates and with faulty sensor outputs. Under such circumstances, both linear and MLP non-adaptive classifiers fail immediately. The CRBM classifier and training approach proposed in this paper recovers from major, nonlinear drift within a short period of time. This is particularly useful and important in the chemical sensing application that forms the motivation for this study, where robustness in the face of miniature, noisy and unreliable sensors is the primary challenge. The work in this paper reinforces a more general capability, that a suitable generative model with a constrained training process can adapt to at least some environmental changes such as sensor drift while presenting a consistent (effectively autonomously recalibrated) representation of drifting data to subsequent layer(s) of processing.

Although the proposed classifier has only been applied to clusters that are only modestly nonlinear, its extension to completely nonlinear data distributions is also possible and has been examined in the context of artifical data. This research work is currently still in progress and will be reported in a subsequent paper with real data.

\section{Acknowledgments}

The authors are grateful to Dr. Erik Johannessen, Dr. David Cumming, Professor Jon Cooper and collaborators at Glasgow University for providing the sensor data. This project is supported by Scottish Higher Education Funding Council (Grant Number: RDG 130) and EPSRC (GR/ R47318).

\section{References}

1 Aguilo, J., Millan, J., and Villa, R.: 'Micro and nano technologies in medical applications: a challenge'. Proceedings of the International Semiconductor Conference, Sinaia, Romania, 2001, Vol. 1, pp. 247-255

2 Given Imaging Ltd, http://www.givenimaging.com, 2001

3 Zhou, G.X.: 'Swallowable or implantable body temperature telemeter - body temperature radio pill'. Proceedings of 15th Annual Northeast Bioengineering Conference, Boston, MA, USA, 1989, pp. 165-166

4 Evans, D.F., Pye, G., Bramley, R., Clark, A.G., Dyson, T.J., and Hardcastle, J.D.: 'Measurement of gastrointestinal $\mathrm{pH}$ profiles in normal ambulant human subjects', Gut, 1988, 29, pp. 1035-1041

5 Jobst, G., Urban, G., Jachimowicz, A., Kohl, F., Tilado, O., Lettenbichler, I., and Nauer, G.: 'Thin film Clark-type oxygen sensor based on novel polymer membrane systems for in vivo and biosensor applications', Biosens. Bioelectron., 1993, 8, pp. 123-128

6 Mackay, S.: 'Radio telemetering from within the body', Science, 1961, 134, pp. 1196-1202

7 Murray, A.F., and Woodburn, R.J.: 'The prospects for analogue neural VLSI', Int. J. Neural Syst., 1998, 8, (5), pp. 559-580

8 Johannessen, E.A., Wang, L., Cui, L., Tang, T.B., Ahmadian, M., Astaras, A., Reid, S.W., Yam, P., Murray, A.F., Flynn, B.W., Beaumont, S.P., Cumming, D.R.S., and Cooper, J.M.: 'Implementation of distributed sensors in a microsystems format', IEEE Trans. Biomedical Eng., 2003, (in press)

9 Ackley, D.H., Hinton, G.E., and Sejnowski, T.J.: 'A learning algorithm for Boltzmann machine', Cogn. Sci., 1985, 9, pp. 147-169

10 Chen, H., and Murray, A.F.: 'A continuous restricted Boltzmann machine with a hardware-amenable learning algorithm'. Proceedings for the 12th International Conference on Artificial Neural Networks, Madrid, Spain, 2002, pp. 358-363

11 Hinton, G.E.: 'Training products of experts by minimizing contrastive divergence', Neural Comput., 2002, 14, (8), pp. 1771-1800

12 Murray, A.F.: 'Novelty detection using products of simple experts - a potential architecture for embedded systems', Neural Netw., 2001, 14, pp. 1257-1264

13 Rashid, M.H.: 'Microelectronics circuit analysis and design' (PWS Publishing Company, Boston, MA, USA, 1999)

14 Bergveld, P.: 'Development, operation and application of the ionsensitive field effect transistor as a tool for electrophysiology', IEEE Trans. Biomed. Eng., 1972, 19, pp. 342-351

15 Rasmussen, C.E., and Ghahramani, Z.: 'Occam's razor', Adv. Neural Inf. Process. Syst., 2001, 13, pp. 294-300

16 Widrow, B., and Hoff, M.E.: ‘Adaptive switching circuits'. WESCON Convention Record, 1960, Vol. IV, pp. 96-104

17 Tang, T.B., Johannessen, E., Wang, L., Astaras, A., Ahmadian, M., Murray, A.F., Cooper, J.M., Beaumont, S.P., Flynn, B.W., and Cumming, D.R.S.: 'Towards a miniature wireless integrated multisensor microsystem for industrial and biomedical applications', IEEE Sens. J. Special Issue on Integrated Multisensor Systems and Signal Processing, 2002, 2, (6), pp. 628-635

18 Amari, S.: 'A theory of adaptive pattern classifiers', IEEE Trans. Electron. Comput., 1967, 16, (3), pp. 299-307

19 Barkai, N., Seung, H.S., and Sompolinsky, H.: 'On-line learning of dichotomies', Adv. Neural Inf. Process. Syst., 1994, 7, pp. 303-310

20 Murata, N., Muller, K., Ziehe, A., and Amari, S.: 'Adaptive on-line learning in changing environments', Adv. Neural Inf. Process. Syst., 1996,9 , pp. $599-605$

21 Hagan, M.T., Demuth, H.B., and Beale, M.H.: 'Neural network design' (PWS Publishing, Boston, MA, USA, 1996) 